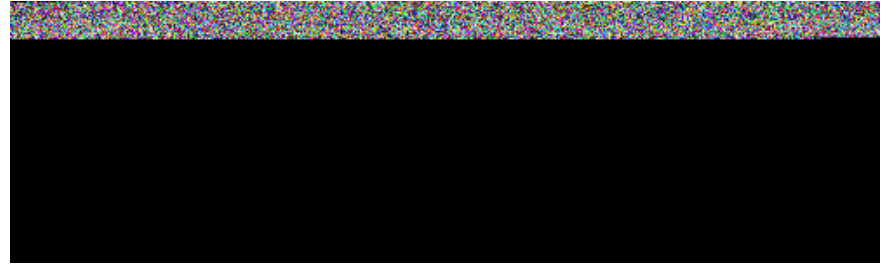

This information is current as of April 26, 2023.

\title{
Accumulation of Brain Hypointense Foci on Susceptibility-Weighted Imaging in Childhood Ataxia Telangiectasia
}

R.A. Dineen, C.V. Blanchard, S. Pszczolkowski, S. Paine, M. Prasad, G. Chow, W.P.

Whitehouse and D.P. Auer

AJNR Am J Neuroradiol 2021, 42 (6) 1144-1150

doi: https://doi.org/10.3174/ajnr.A7107

http://www.ajnr.org/content/42/6/1144 


\title{
Accumulation of Brain Hypointense Foci on Susceptibility- Weighted Imaging in Childhood Ataxia Telangiectasia
}

\author{
(D) R.A. Dineen, (D) C.V. Blanchard, (D) S. Pszczolkowski, (D). Paine, (D) M. Prasad, (D) G. Chow, (D)W.P. Whitehouse, and (DD.P. Auer
} O- $\equiv$

\begin{abstract}
BACKGROUND AND PURPOSE: SWI hypointense cerebral lesions have been reported in adults with the inherited cerebellar neurodegenerative disorder ataxia telangiectasia. This study aims to establish the prevalence, age-dependency, and spatial distribution of these lesions in children and young people with ataxia telangiectasia.
\end{abstract}

MATERIALS AND METHODS: Participants with classic ataxia telangiectasia and matched controls underwent SWI acquisition at $3 T$ at 1 or
2 time points. SWI hypointense lesions were manually labeled according to the Microbleed Anatomical Rating Scale. Differences in preva-
lence of lesion number between groups with ataxia telangiectasia and without ataxia telangiectasia were tested with the Fisher exact
test, and differences in age between participants with ataxia telangiectasia with and without lesions were tested using independent sam-
ples Mann-Whitney $U$ test. The relationship between age and lesion number was modeled as an exponential function.

RESULTS: Analyzable SWI datasets from 17 participants with ataxia telangiectasia (with median age at first scan of 12.4 years; range, 4.620.2 years; 8 [47\%] were female) and 22 matched healthy controls showed prevalence of SWI hypointense lesions in $41 \%$ of participants with ataxia telangiectasia and $0 \%$ in controls $(P=.001$, Fisher exact test). Lesions were exclusively supratentorial and predominantly lobar. Participants with ataxia telangiectasia with SWI hypointense lesions were older than those without (median age 5.2 years versus 9.3 years, $\mathrm{U}=10.5, P=.014)$. An exponential curve described the relationship between age and lesion number $\left(R^{2}=0.67\right)$.

CONCLUSIONS: SWI hypointense lesions are common in children and young people with ataxia telangiectasia, accumulating from 12 years of age onward. In contrast to cerebellar-dominant neurodegeneration in ataxia telangiectasia, SWI hypointense lesions were exclusively supratentorial. Further investigation is needed to establish the clinical relevance of these imaging-detected lesions.

ABBREVIATIONS: A-T = ataxia telangiectasia; ATM = ataxia-telangiectasia mutated; CATNAP $=$ Childhood A-T Neuroimaging Assessment Project; SWI $=$ susceptibility weighted imaging

A taxia telangiectasia (A-T, Online Mendelian Inheritance in Man, No. 208900) is an autosomal recessive multisystem disorder associated with cerebellar neurodegeneration, telangiectasia (particularly conjunctival), immunodeficiency, pulmonary disease, radiation sensitivity, and cancer predisposition. ${ }^{1}$ People with "classical" A-T have either no production of the ataxia-telangiectasia mutated (ATM) protein or produce completely nonfunctioning ATM protein. People who produce a significantly reduced amount of functioning ATM have a milder variant of A-T. ${ }^{2}$

Received November 16, 2020; accepted after revision December 24.

From the Radiological Sciences, Division of Clinical Neuroscience (R.A.D., C.V.B., S. Pszczolkowski, D.P.A.), Sir Peter Mansfield Imaging Centre (R.A.D., D.P.A.), and Division of Child Health (W.P.W.), University of Nottingham, Nottingham, England; National Institute for Health Research Nottingham Biomedical Research Centre (D.P.A.), Nottingham, England; and Department of Pathology (S. Paine) and Nottingham Children's Hospital (M.P., G.C., W.P.W.), Nottingham University Hospitals National Health Service Trust, Nottingham, England.

The funding for the research was from research grants awarded jointly by A-T Children's Project and Action for A-T (grant reference: "The CATNAP Study"), and jointly by Action for A-T and BraShA-T (grant reference: 17NOT03).
Cerebellar atrophy is the dominant neuroimaging finding in A-T (reviewed in detail by Sahama et $\mathrm{al}^{3}$ ), but several case series and individual case reports describe hypointense foci in the brain parenchyma using SWI or T2WI. In a series of 10 adults with A-T, 19-34 years of age, Lin et $\mathrm{al}^{4}$ observed hypointense foci using SWI in 7 (70\%) of the participants. These ranged from a single hypointense focus in a 21-year-old through to "innumerable" foci in individuals with 28 and 34 years of age. Wallis et $\mathrm{al}^{5}$ presented a series of 12 adults with A-T, 23-47 years of age, 4 of whom had hypointense foci (solitary in 2, multiple in 2 ) in the

The funders had no role in the study design; the collection, analysis, and interpretation of data; the writing of the report; and the decision to submit the article for publication.

Please address correspondence to Robert A. Dineen, PhD, Room A39i, Medical School, Queen's Medical Center, Derby Road, Nottingham, NG7 2UH, United Kingdom; e-mail: rob.dineen@nottingham.ac.uk

- Indicates open access to non-subscribers at www.ajnr.org

三 Indicates article with online supplemental data.

http://dx.doi.org/10.3174/ajnr.A7107 
brain parenchyma on T2WI. Further examples are provided by Liu et $\mathrm{al}^{6}$ (27-year-old man with multiple hypointense foci on SWI) and Ciemins and Horowitz ${ }^{7}$ (31-year-old woman with multiple hypointense foci on both T1- and T2WI).

To date, the prevalence, age distribution, and spatial distribution of SWI hypointense foci in the brains of children with A-T has not been investigated. To address this, we used data from the Childhood A-T Neuroimaging Assessment Project (CATNAP $^{8}$ to test the hypothesis that SWI hypointensities accumulate during childhood in children and young people with classical A-T.

\section{MATERIALS AND METHODS}

The data for this analysis are from the first and second phases of CATNAP. In brief, the first phase of CATNAP involved the acquisition of multiparametric MR imaging and neurologic data from children and young people 6-18 years of age with A-T and matched healthy controls. As described previously, ${ }^{8}$ participants with A-T were recruited from the UK National Pediatric A-T Clinic and were excluded if they had contraindication to MR imaging, concurrent or previous cancer or cancer treatment, or other non-A-T neurologic or neurosurgical conditions. Healthy volunteers were recruited from local community groups and excluded if they had contraindications to MR imaging or any neurologic, neurosurgical, or other medical conditions. Recruitment ran from January 2015 to September 2016. The results of volumetric analysis and spectroscopy have been reported. ${ }^{8}$ The second phase of CATNAP involved invitation of previous participants for repeat multiparametric MR imaging on the same scanner using an identical protocol after an interval of 2-4 years, and recruitment of new participants 3-6 years of age to test the feasibility of the data acquisition in very young children. Informed consent was obtained from participants who were 1618 years of age and from parents/guardians of participants under the age of 16 years of age. The first and second phases of CATNAP were approved by UK National Research Ethics Service (references 14/EM/1175 and 18/SW/0078, respectively).

\section{Image Acquisition and Analysis}

All participants underwent $\mathrm{MR}$ imaging scanning on a $3 \mathrm{D}$ Discovery MR750 (GE Healthcare) with a 32-channel head coil, without sedation. In addition to standard pediatric MR imaging preparation, younger participants were shown an animation to prepare them for MR imaging ${ }^{9}$ and were able to watch video content during the scan on a monitor compatible with MR imaging. The full MR imaging protocol is detailed in the Online Supplemental Data. The results presented here relate to the axial SWI acquisition performed using the $\mathrm{T} 2{ }^{*}$ weighted angiography susceptibility-based 3D multiecho gradient sequence (216 slices, section thickness $=1 \mathrm{~mm}$ with $0.5 \mathrm{~mm}$ between slices, $\mathrm{FOV}=256 \times 256, \mathrm{TR}=39.3 \mathrm{~ms}$, effective $\mathrm{TE}=24.68 \mathrm{~ms}$, flip angle $=15^{\circ}$, scan duration $=4: 19$ minutes).

Two experienced neuroradiologists (R.A.D., D.P.A.), who were unaware of disease status, independently labeled all hypointensities manually on SWI according to the anatomic classification specified in the Microbleed Anatomical Rating Scale ${ }^{10}$ using the paintbrush drawing tool in ITK-SNAP software (http://www. itksnap.org). ${ }^{11}$ After the independent review, any discrepancies in lesion labeling were resolved through discussion leading to consensus.

\section{Statistical Analysis}

Comparison of age distribution between the groups with A-T and without A-T was performed using the independent samples Mann-Whitney U test. Prevalence of SWI hypointense lesions (using the second scan for those with 2 scans) was expressed as a percentage with $95 \%$ CI for each group, and differences in prevalence of SWI hypointense lesions between groups with A-T and without A-T were tested using the Fisher exact test. Within the group with A-T, differences in age distribution between those with and without SWI hypointense lesions was determined using the independent samples Mann-Whitney $U$ test, using age at second scan for those with 2 scans. Exact significance (2-tailed) was calculated, and the significance level was set at $\alpha<.05$. We modeled the relationship between age and observed number of SWI hypointense lesions as an exponential function $y=a \cdot e^{b \cdot x}$, where $x$ is age, $y$ is the observed number of lesions, and $a$ and $b$ are unknown parameters. To find the values of $a$ and $b$ that best fitted the data, we ran a trustregion reflective optimization ${ }^{12,13}$ with a starting point of $a=0.01, \quad b=0.5$.

\section{RESULTS}

Twenty-two children and young people with A-T and 22 without A-T underwent at least 1 SWI acquisition. Of these, 8 with A-T and 13 without A-T had a second SWI scan. In total, 13 of the SWI datasets (10 from the group with A-T and 3 from the group without A-T group) were excluded from the analysis because of significant participant motion artifacts. One scan where the image quality was felt to be borderline showed a clear parenchymal SWI hypointense lesion, and this scan was retained in the analysis. The analyzed images were from 17 children and young people with A-T (with median age at first scan of 12.4 years; range, 4.6-20.2 years; 8 [47\%] were female) and 22 without A-T (median age at first scan was 13.0 years; range, $5.5-17.8$ years; 11 [50\%] were female). There was no group difference in age distribution $(U=188$, exact $P=.977)$. Analyzable second SWI scans were available from 2 people in the group with A-T (interscan intervals of 3.3 and 3.5 years) and from 10 in the group without A-T (median interscan interval of 2.4 years, range, 2.3-3.5 years). Of the 17 participants with A-T included in this report, 8 had no ATM expression, 8 had ATM expression but no kinase activity, and 1 had ATM expression with some residual kinase. No participant with A-T reported (or had parental/guardian report of) an alternative cause for cerebral microbleeds, such as history of head trauma, hypertension, or coagulopathy. Sixteen of the participants with A-T and 21 of the participants without A-T were included in our previous publication relating to cerebellar volumetry, diffusion, and spectroscopy. ${ }^{8}$ Recruitment to CATNAP and CATNAP-2 and the availability of acceptable-quality SWI data are summarized in the flowchart (Online Supplemental Data). 


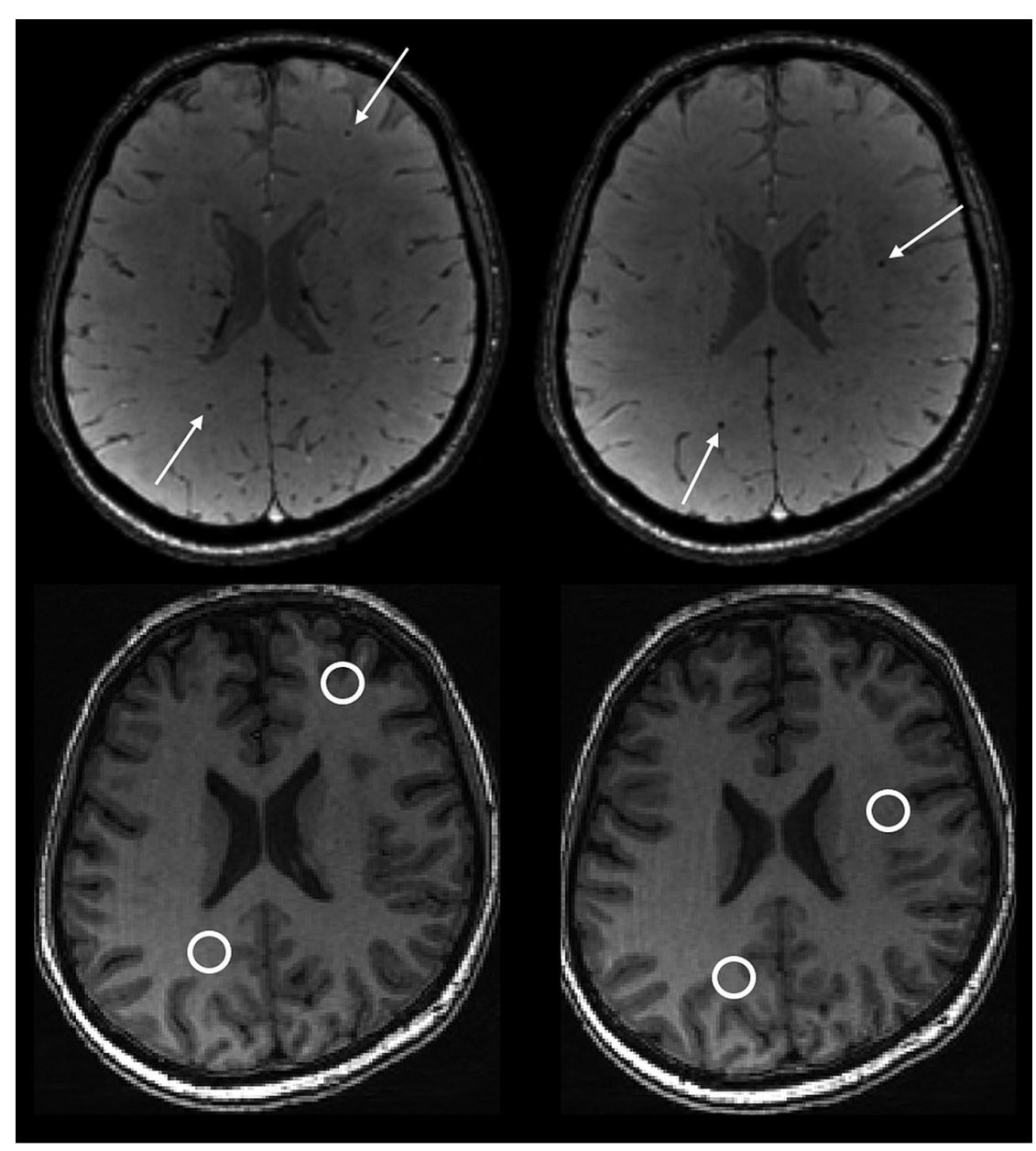

FIG 1. Upper row: Example of parenchymal SWI hypointense lesions in a participant with A-T aged 17.8years. Axial images showing 4 separate lesions (white arrows). A total of 23 similar lesions (not all shown) were present on the imaged volume. Lower row: Corresponding axial fast-spoiled gradient-recalled images showing that the lesions are not clearly visible on the T1weighted volumetric acquisition.

9.3 years [range, $4.6-17.6$ years], respectively, $U=10.5, P=.014$; Fig 4$)$. No lesions were identified in children with A-T younger than 12 years of age. The 2 participants with A-T who had analyzable scans at 2 time points both showed an increase in the number of lesions between the first and second scans (Figs 2 and 5). The curve fitted for the number of SWI hypointense lesions against age for the A-T group (Fig 2) had $R^{2}=0.67$ and was described by the equation:

number of lesions

$$
=0.00168 \cdot e^{0.4841 \cdot \text { age }}
$$

The $95 \%$ confidence limits for coefficient $a$ are -0.00917 and 0.01252 , and for coefficient $b$ are 0.1575 and 0.8106 .

\section{DISCUSSION}

In our cohort of children and young people with A-T, we identified a prevalence of SWI hypointense lesions of $41 \%$. In comparison, no such lesions were identified in any participants of the well-matched control group. Furthermore, despite the limited number of data points in the group with $\mathrm{A}-\mathrm{T}$, we demonstrate a clear relationship between the number of SWI hypointense lesions and age across the group, as well as direct evidence on new lesion accumulation in the 2 participants with A-T who had analyzable SWI at 2 time points.

SWI hypointense lesions were present in 7 of 17 participants with $\mathrm{A}-\mathrm{T}$ on at least 1 scan, giving a prevalence of $41 \%$ (95\% CI, $22 \%-64 \%$ ) (Fig 1). For the 7 participants with A-T who had SWI hypointense lesions, the number of lesions ranged from 1 to 41 $($ median $=3$ ) (Fig 2). Lesions ranged in size from $2 \mathrm{~mm}$ (the lower limit for inclusion) up to $4 \mathrm{~mm} .{ }^{10}$ No lesions were identified in any participants in the group without A-T, 0/22 (95\% CI, $0 \%-18 \%)$. The prevalence of lesions differed significantly between the groups with A-T and without A-T $(P=.001$, the Fisher exact test). Anatomic distribution of SWI hypointense lesions in the group with A-T is shown in the Table and Fig 3. No SWI hypointense lesions were found in the cerebellum and brain stem, and supratentorial lesions were overwhelmingly lobar rather than deep. The SWI hypointense lesions were not clearly visible on the T1-weighted fast-spoiled gradient-recalled images (Fig 1, bottom row).

A significant age difference was identified between participants with A-T who did and did not have SWI hypointense lesions (median age was 15.2 years [range, 12.4-20.2 years] versus
Previous case reports and series have reported SWI hypointense lesions in adults with $\mathrm{A}-\mathrm{T}$, but the prevalence and natural history of these lesions in children with A-T were not known. Indeed, Lin et $\mathrm{al}^{4}$ state that in their clinical experience, SWI hypointense lesions are absent in children with A-T; and that lesions are acquired and only cross a threshold of detectability in early adulthood. Our findings support the notion that lesions are acquired in an age-related manner but counter the statement relating to absence in childhood. It is possible that improvements in acquisition of SWI since the time of that publication in 2014 allow us to visualize lesions more clearly.

The nature and significance of these imaging-detected lesions remain uncertain. Histopathologic studies have demonstrated the presence of a distinctive vascular abnormality in people with A-T, referred to as "gliovascular nodules." "14 These consist of dilated capillary loops, often containing fibrin thrombi, around which is perivascular hemorrhage and hemosiderin deposition, with associated demyelination and astrocytic gliosis, including atypical forms (some of 

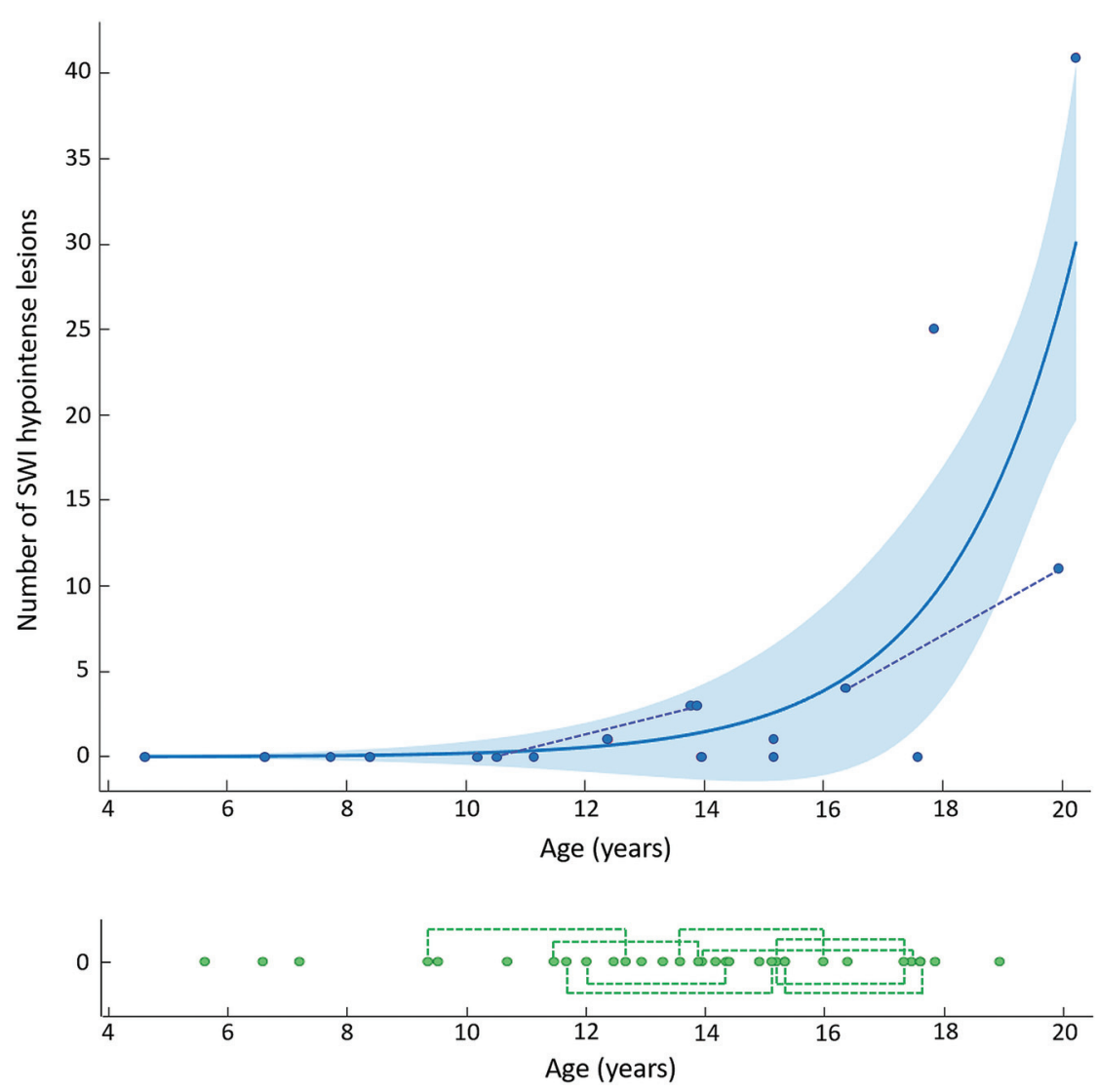

FIG 2. Scatterplots showing relationship between age and number of lesions in people with A-T (upper chart, blue) and people without A-T (lower chart, green). Dotted lines indicate pairs of scans performed in the same individual. The curved blue line indicates the exponential model fitted to the data, with the blue shaded area indicating the $95 \%$ confidence intervals.

Anatomic distribution of SWI hypointense lesions in the group with A-T

\begin{tabular}{lcc}
\hline & \multicolumn{2}{c}{ Participants with A-T $(\boldsymbol{n}=17)$} \\
\cline { 2 - 3 } & $\begin{array}{l}\text { Number of Subjects } \\
\text { with Lesions }\end{array}$ & $\begin{array}{l}\text { Total Number } \\
\text { of Lesions }\end{array}$ \\
\hline Lobar & $6(35 \%)$ & 82 \\
Deep Gray Matter & $4(24 \%)$ & 7 \\
Cerebellum & $0(0 \%)$ & 0 \\
Brain Stem & $0(0 \%)$ & 0 \\
All Sites & $7(41 \%)$ & 89 \\
\hline
\end{tabular}

which contain eosinophilic cytoplasmic inclusions) in the adjacent parenchyma. ${ }^{15,16}$ It is likely that the focal hypointensities detected in our work and by previous studies are the in vivo imaging correlation of these gliovascular nodules, though direct correlation between imaging and histopathology has not been performed to our knowledge. Notably, pathologic studies show that the distribution of gliovascular nodules is predominantly in the cerebral white matter, occasionally in the basal ganglia and cerebral cortex, but not in the cerebellum. This description of location matches closely the distribution of lesions found in our data. The reason for the observed distribution of lesions is not known, nor why the posterior fossa appears to be spared. There may be underlying factors related to vascular structure and/or vascular expression of different molecules or receptors, or there may be a relationship to hemodynamic parameters. Combined imaging, histopathologic studies, and molecular studies may help to clarify the underlying causes of the formation and distribution of the lesions.

However, it is also possible that pathologic changes other than gliovascular nodules could account for the imaging appearances. A histopathologic case report described hyalinization of cerebral blood vessels associated with intimal and adventitial hypertrophy in a patient with advanced disease, ${ }^{17}$ indicating the presence of cerebrovascular degenerative pathology that is separate from the gliovascular nodules. However, the limited available reports do not suggest that this cerebrovascular hyalinization occurring with A-T is directly associated with localized microhemorrhage or hemosiderin deposition detected by SWI. Lin at $\mathrm{al}^{4}$ comment that CNS irradiation can cause similar appearances on SWI because of the development of radiation-induced cavernous hemangiomas, which may be a late sequela of cancer treatment. ${ }^{18}$ This may be relevant because $A-T$ is associated with an increased cellular sensitivity to ionizing radiation ${ }^{19}$ and abnormal DNA damage response. However, the histopathologic description of vascular changes seen in A-T do not closely overlap with those described for radiation-induced cavernous hemangiomas, which appear to show 2 distinct histologic patterns: those resembling typical cerebral cavernous hemangiomas, and those caused by radiation-induced fibrinoid vascular necrosis and vascular leakage. ${ }^{20}$

It is currently unclear whether the presence of the hypointense lesions on SWI in people with A-T confers an increased risk of intracerebral hemorrhage. In other conditions with similarappearing lesions on SWI, such as cerebral amyloid angiopathy and cerebral cavernous hemangiomas, the presence of lesions indicates an increased macrohemorrhage risk. ${ }^{21,22}$ There are only 2 reports of macrohemorrhage in people with $\mathrm{A}-\mathrm{T},{ }^{23,24}$ but the relationship to any underlying SWI hypointensities has not been explored.

In other regards, the relationship between presence or number of SWI hypointense lesions and other clinical or imaging manifestations of A-T is also not known. Of particular interest is the question of whether SWI hypointense lesions could have an impact on cognitive function. Previous studies have identified cognitive deficits in people with A-T, including in the domains of language, processing speed, visuospatial processing, working memory, attention, and abstract reasoning, ${ }^{25,26}$ which have been 


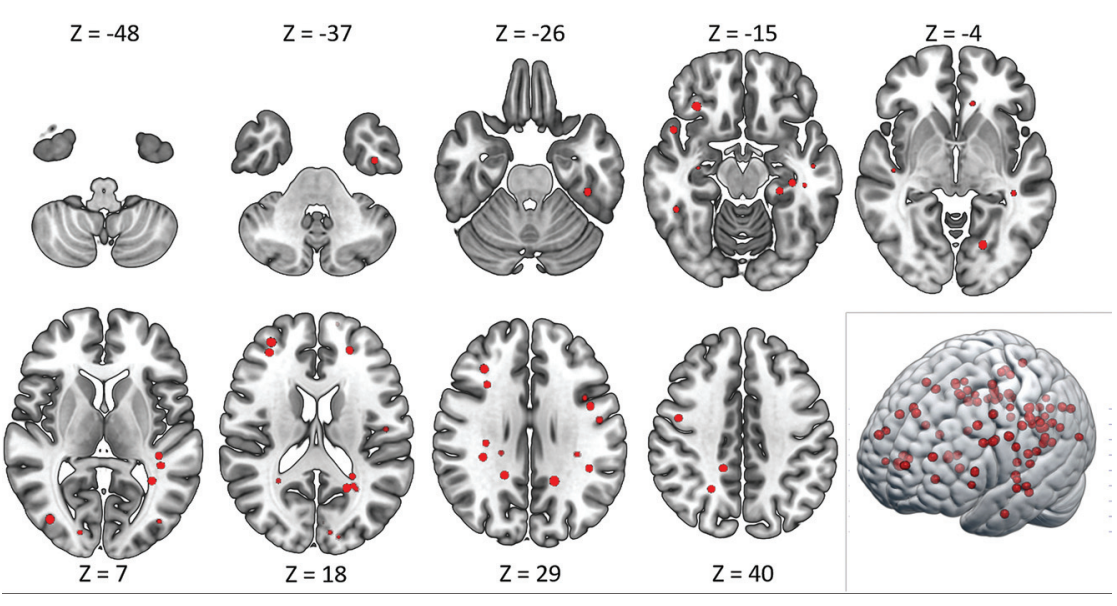

FIG 3. Distribution of SWI hypointense lesions. Representative axial images from the summated SWI hypointense lesion maps from all participants with A-T, displayed on a Montreal Neurological Institute 152 template. Axial section locations indicated by the z-axis coordinate. Note the absence of cerebellar or brain stem lesions. Lower right image: The same summated lesion map data displayed as a 3D rendered image.

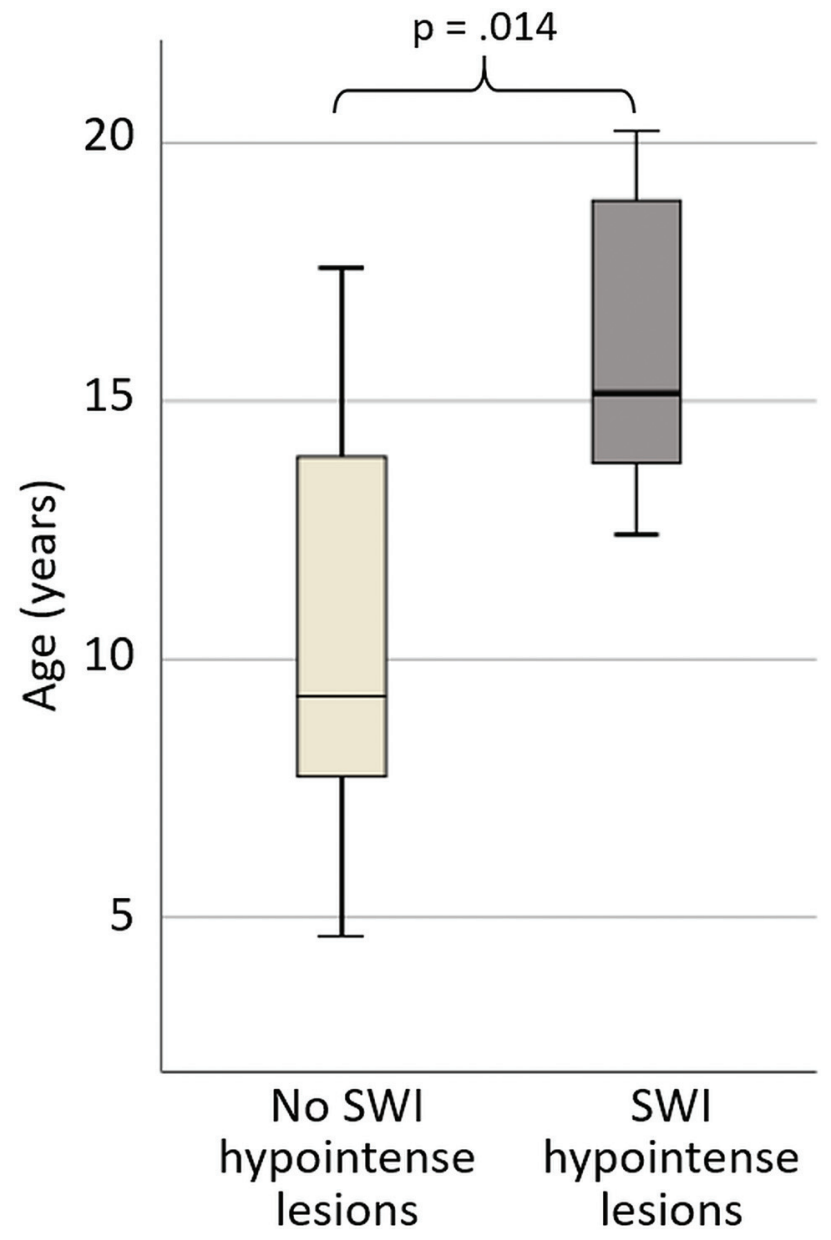

FIG 4. Boxplot showing age distribution for A-T participants without (light gray) and with (dark gray) SWI hypointense lesions.

attributed to the cerebellar cognitive affective syndrome. ${ }^{27}$ Given that other diseases associated with multifocal cerebral white matter lesions, such as multiple sclerosis ${ }^{28}$ and small-vessel disease, ${ }^{29}$ are associated with cognitive dysfunction, an impact of cerebral
SWI hypointense lesions on cognition in people with $\mathrm{A}-\mathrm{T}$ is possible. The small number of participants with A-T showing SWI hypointense lesions in our dataset limits further exploration of the relationship between the presence or number of these lesions and other neurologic, cognitive, or imaging metrics. Ideally future, larger imaging studies in A-T will include SWI acquisitions, allowing the clinical relevance of these lesions to be formally investigated.

This study is the largest reported series of SWI in childhood A-T, and has a well-matched control group, but is still limited by small sample size. There was a high rate of data rejection in the group with A-T (one-third of the SWI datasets), because of excessive participant motion rendering the image unreliable for analysis, which reduced the number of longitudinal SWI datasets in the group with A-T from 8 to 2 . We used careful participant preparation and carefully immobilized the participant's head in the head coil using inflatable pads. However, A-T is a movement disorder and those affected are prone to involuntary movements. In addition, the SWI acquisition was toward the end of a multisequence research MR imaging protocol, which is likely to have affected tolerance and led to restlessness. We have taken steps to reduce observer bias during the identification of SWI hypointense lesions, which was performed independently by 2 experienced neuroradiologists who were not aware of the disease status. However, people with A-T typically have overt cerebellar atrophy that would have been visible on the SWI datasets during the labeling of SWI hypointense lesions and it is possible that the reviewing neuroradiologists may have unintentionally recognized that a scan was from a participant with A-T.

\section{CONCLUSIONS}

This work demonstrates that SWI hypointense lesions are present in children and young people with A-T with a prevalence of $41 \%$ and accumulation is shown across the childhood age range. No lesions were seen in children younger than 12 years of age. Furthermore, in our cohort, lesions were exclusively supratentorial in an overwhelmingly lobar distribution, which is notable because the burden of neurodegeneration in people with A-T occurs in the cerebellum. ${ }^{3}$ Further investigation is needed to elucidate the nature and relevance of these imaging-detected lesions.

\section{ACKNOWLEDGMENTS}

The authors would like to thank the children, young people, and families who participated in the CATNAP studies. We also gratefully acknowledge the support Kay Atkins, Anne Murray, and William Davis and at the A-T Society for support with publicizing 


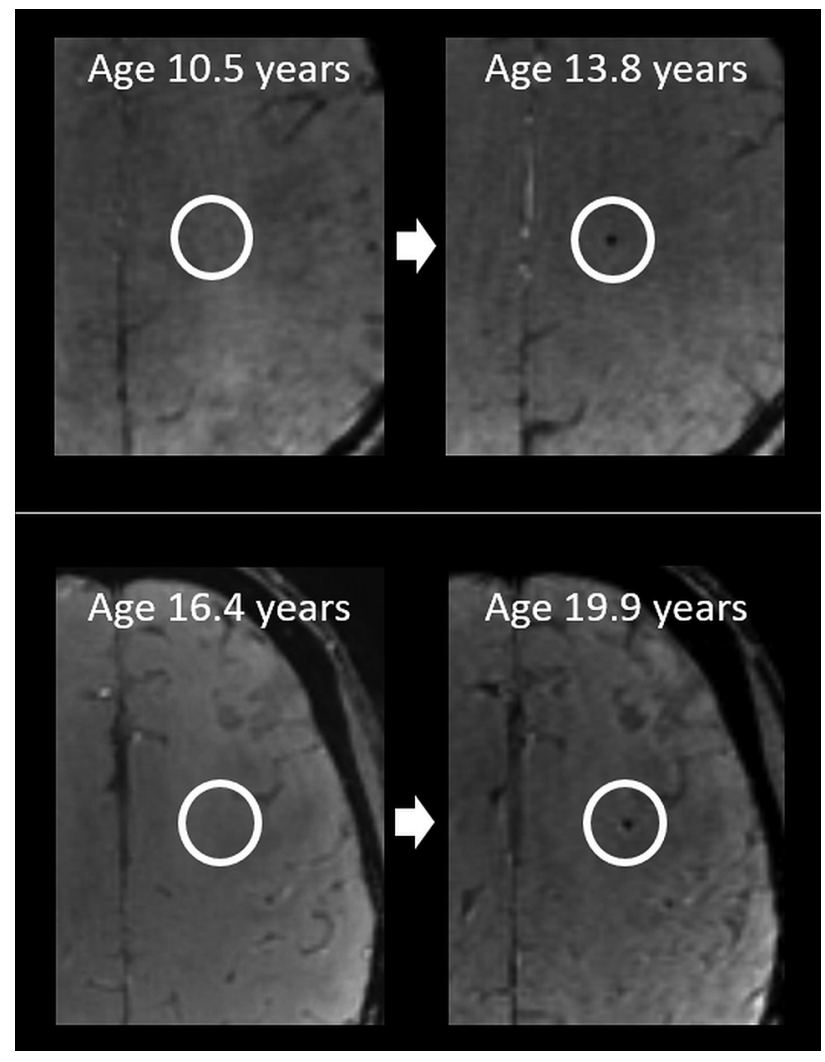

FIG 5. Examples of new lesions in participants with A-T who had scans at 2 time points. Upper and lower rows indicate different participants; ages indicated on each image.

the study and arranging schedules and transport for participant visits; Dr Min Ong and Dr Ouliana Panagioti for additional support with neurologic assessments; $\mathrm{Mr}$ Andrew Cooper for performing the MR imaging scans; Mrs Catherine Gibney for undertaking play preparation with younger participants; and $\mathrm{Dr}$ Felix Raschke, Ms Hannah McGlashan, and Dr Jeyanthi Rangaraj for recruitment and additional participant assessments.

Disclosures: Robert A. Dineen-RELATED: Grant: charity funding. Caroline V. Blanchard-RELATED: Grant: charity funding. Stefan Pszczolkowski-RELATED: Grant: charity funding. Gabriel Chow-RELATED: Grant: charity funding. William Whitehouse -RELATED: Grant: charity funding, UNRELATED: Consultancy: pharma company advice, Comments: Eli Lilly, expert meeting to discuss a new migraine drug trial. No relevance to the submitted study; GW Pharmaceuticals, expert meeting to discuss the marketing authorization of a new antiepileptic drug. No relevance to the submitted study. Dorothee P. Auer-RELATED: Grant: charity funding; UNRELATED: Grants/ Grants Pending: National Institute for Health Research, Versus Arthritis, Parkinson's UK, Michael J. Fox Foundation, Biogen*; Travel/Accommodations/Meeting Expenses Unrelated to Activities Listed: German and Irish National Research Foundations, Comments: Reimbursement of travel/accommodation for grant review panel meeting*.

\section{REFERENCES}

1. Rothblum-Oviatt C, Wright J, Lefton-Greif MA, et al. Ataxia telangiectasia: a review. Orphanet J Rare Dis 2016;11:159 CrossRef Medline

2. Schon K, van Os NJ, Oscroft N, et al. Genotype, extrapyramidal features, and severity of variant ataxia-telangiectasia. Ann Neurol 2019;85:170-80 CrossRef Medline

3. Sahama I, Sinclair K, Pannek K, et al. Radiological imaging in ataxia telangiectasia: a review. Cerebellum 2014;13:521-30 CrossRef Medline
4. Lin DD, Barker PB, Lederman HM, et al. Cerebral abnormalities in adults with ataxia-telangiectasia. AJNR Am J Neuroradiol 2014;35:119-23 CrossRef Medline

5. Wallis LI, Griffiths PD, Ritchie SJ, et al. Proton spectroscopy and imaging at $3 \mathrm{~T}$ in ataxia-telangiectasia. AJNR Am J Neuroradiol 2007;28:79-83 Medline

6. Liu HS, Chen YC, Chen CY. Cerebral microbleeds and iron depletion of dentate nuclei in ataxia-telangiectasia. Neurology 2016;87:1062-63 CrossRef Medline

7. Ciemins JJ, Horowitz AL. Abnormal white matter signal in ataxia telangiectasia. AJNR Am J Neuroradiol 2000;21:148385 Medline

8. Dineen RA, Raschke F, McGlashan HL, et al. Multiparametric cerebellar imaging and clinical phenotype in childhood ataxia telangiectasia. Neuroimage Clin 2020;25:102110 CrossRef Medline

9. McGlashan HL, Dineen RA, Szeszak S, et al. Evaluation of an internet-based animated preparatory video for children undergoing nonsedated MRI. Br J Radiol 2018;91:20170719 CrossRef Medline

10. Gregoire SM, Chaudhary UJ, Brown MM, et al. The Microbleed Anatomical Rating Scale (MARS): reliability of a tool to map brain microbleeds. Neurology 2009;73:1759-66 CrossRef Medline

11. Yushkevich PA, Piven J, Hazlett HC, et al. User-guided 3D active contour segmentation of anatomical structures: significantly improved efficiency and reliability. Neuroimage 2006;31:1116-28 CrossRef Medline

12. Branch MA, Coleman TF, Li Y. A subspace, interior, and conjugate gradient method for large-scale bound-constrained minimization problems. SIAM J Sci Comput 1999;21:1-23 CrossRef

13. Coleman $\mathrm{TF}, \mathrm{Li} \mathrm{Y}$. On the convergence of interior-reflective Newton methods for nonlinear minimization subject to bounds. Mathematical Programming 1994;67:189-224 CrossRef

14. Boder EA. Telangiectasia. In: Gomez MR, ed. Neurocutaneous Disorders. Butterworth Publishers; 1987: 95-117

15. Amromin GD, Boder E, Teplitz R. Ataxia-telangiectasia with a 32 year survival. a clinicopathological report. J Neuropathol Exp Neurol 1979;38:621-43 CrossRef Medline

16. Agamanolis DP, Greenstein JI. Ataxia-telangiectasia. Report of a case with Lewy bodies and vascular abnormalities within cerebral tissue. J Neuropathol Exp Neurol 1979;38:475-89 CrossRef Medline

17. Kamiya M, Yamanouchi H, Yoshida T, et al. Ataxia telangiectasia with vascular abnormalities in the brain parenchyma: report of an autopsy case and literature review. Pathol Int 2001;51:271-76 CrossRef Medline

18. Jain R, Robertson PL, Gandhi D, et al. Radiation-induced cavernomas of the brain. AJNR Am J Neuroradiol 2005;26:1158-62 Medline

19. Huo YK, Wang Z, Hong JH, et al. Radiosensitivity of ataxia-telangiectasia, $\mathrm{X}$-linked agammaglobulinemia, and related syndromes using a modified colony survival assay. Cancer Res 1994;54:2544-47 Medline

20. Kleinschmidt-DeMasters BK, Lillehei KO. Radiation-induced cerebral vascular "malformations" at biopsy. J Neuropathol Exp Neurol 2016;75:1081-92 CrossRef Medline

21. Charidimou A, Imaizumi T, Moulin S, et al. Brain hemorrhage recurrence, small vessel disease type, and cerebral microbleeds: a meta-analysis. Neurology 2017;89:820-29 CrossRef Medline

22. Kearns KN, Chen CJ, Yagmurlu $\mathrm{K}$, et al. Hemorrhage risk of untreated isolated cerebral cavernous malformations. World Neurosurg 2019;131:e557-61 CrossRef Medline

23. Casaril M, Gabrielli GB, Capra F, et al. Ataxia telangiectasia. Description of a case with multiple cerebral hemorrhages and liver cirrhosis. Minerva Med 1982;73:2183-88 Medline

24. Nardelli E, Fincati E, Casaril M, et al. Multiple cerebral hemorrhages in ataxia-telangiectasia. A case report. Acta Neurol (Napoli) 1985;7:494-99 Medline

25. Hoche F, Frankenberg E, Rambow J, et al. Cognitive phenotype in ataxia-telangiectasia. Pediatr Neurol 2014;51:297-310 CrossRef Medline 
26. Vinck A, Verhagen MM, Gerven $M$, et al. Cognitive and speech-language performance in children with ataxia telangiectasia. Dev Neurorehabil 2011;14:315-22 CrossRef Medline

27. Hoche F, Daly MP, Chutake YK, et al. The cerebellar cognitive affective syndrome in ataxia-telangiectasia. Cerebellum 2019;18:225-44 CrossRef Medline
28. Dineen RA, Vilisaar J, Hlinka J, et al. Disconnection as a mechanism for cognitive dysfunction in multiple sclerosis. Brain 2009;132:23949 CrossRef Medline

29. Meng D, Hosseini AA, Simpson RJ, et al. Lesion topography and microscopic white matter tract damage contribute to cognitive impairment in symptomatic carotid artery disease. Radiology 2017;282:502-15 CrossRef Medline 